\title{
Fatores de Risco para Doenças Crônicas não Transmissíveis no Brasil: uma Revisão Sistemática
}

\author{
Risk Factors for Chronic non Communicable Diseases in Brazil: a Systematic \\ Review \\ Factores de Riesgo para Enfermedades Crónicas no Transmisibles en Brasil: una \\ Revisión Sistemática
}

\author{
Letícia Casado', Lucia Marques Vianna², Luiz Claudio Santos Thuler ${ }^{3}$
}

\section{Resumo}

No Brasil, devido às mudanças nos perfis demográfico, epidemiológico e nutricional da população e ao controle conseguido em um número de enfermidades transmissíveis, vêm observando-se, nas últimas décadas, uma inversão do perfil epidemiológico com redução das doenças infecciosas e o aumento significativo da prevalência das doenças crônicas não transmissíveis (DCNTs). Essa mudança justifica o estudo dos fatores de risco e determinantes sociais das doenças crônico-degenerativas no país. O presente trabalho teve por objetivo conhecer a prevalência da exposição da população aos principais fatores de risco para o desenvolvimento de DCNTs no Brasil. Foi realizada uma revisão sistemática dos artigos publicados na literatura científica, a partir das bases de dados on-line Lilacs e Medline, entre 2003 e 2008, em português. As prevalências variaram conforme os critérios utilizados e as características das populaçóes estudadas, sendo obtidos os seguintes valores mínimo e máximo para cada um dos fatores de risco estudados: tabagismo $8,7 \%$ a $28,8 \%$, uso abusivo de álcool $0,1 \%$ a $37,7 \%$, excesso de peso $1,5 \%$ a $49,0 \%$, obesidade $9,4 \%$ a $17,6 \%$, sedentarismo $20,1 \%$ a $43,1 \%$, hipertensão arterial $5,3 \%$ a $34,0 \%$, diabetes mellitus $2,7 \%$ a $7,8 \%$. A variação no grau de exposição da população aos fatores de risco presentes nos diversos estudos aponta para a necessidade de padronização dos instrumentos de medida, a fim de que os resultados obtidos nas diferentes localidades possam ser comparados. Além disso, as altas prevalências observadas em algumas áreas indicam a necessidade de intervenções imediatas por meio da implementação de estratégias de prevenção e promoção da saúde dirigidas à redução da exposição da população brasileira aos fatores associados ao risco de desenvolvimento das DCNTs.

Palavras-chave: Doença Crônica; Fatores de Risco; Prevalência; Inquéritos de Morbidade; Estudos Transversais; Brasil

Este trabalho foi vencedor do XIII Prêmio Hélcio Alvarenga, concedido pela Universidade Federal do Estado do Rio de Janeiro (UNIRIO), em dezembro de 2008.

${ }^{1}$ Serviço de Edição e Informação Técnico-Científica da Coordenação de Educação (CEDC) do Instituto Nacional de Câncer (INCA); Mestranda em Neurologia da UNIRIO.

${ }^{2}$ Laboratório de Investigação em Nutrição e Doenças Crônico-Degenerativas (Lindcd) da UNIRIO.

${ }^{3}$ UNIRIO; Pós-Graduação em Oncologia do INCA.

Endereço para correspondência: Letícia Casado - CEDC/INCA - Rua do Resende, 128/ 20 andar - Centro - Rio de Janeiro (RJ), Brasil. CEP: 20231-092. E-mail: leticiac@inca.gov.br 


\section{INTRODUÇÃO}

As transformações sociais e econômicas pelas quais o Brasil vem passando desde o último século têm causado mudanças relevantes no perfil morbimortalidade de nossa população. As doenças infecciosas e parasitárias, principais causas de morte no início do século passado, cederam lugar às doenças crônicas não transmissíveis $(\mathrm{DCNTs})^{1}$. Essa transição epidemiológica tem se refletido na área de saúde pública e o desenvolvimento de estratégias para o controle das DCNTs se tornou uma emergência para o Sistema Único de Saúde (SUS).

As doenças crônicas são definidas como afecções de saúde que acompanham os indivíduos por longo período de tempo, podendo apresentar momentos de piora (episódios agudos) ou melhora sensível ${ }^{2}$. A vigilância epidemiológica (VE) das DCNTs deve reunir um conjunto de açôes que possibilite conhecer sua distribuição, magnitude e tendência de exposição aos seus fatores de risco na população, identificando seus condicionantes sociais, econômicos e ambientais, com o objetivo de subsidiar o planejamento, execução e avaliação das ações de prevenção e controle das mesmas, implementando assim políticas públicas voltadas para a promoção da saúde ${ }^{1}$.

Os fatores de risco para o desenvolvimento das DCNTs vêm sendo classificados como modificáveis ou não modificáveis. Entre os fatores modificáveis, estão a hipertensão arterial, a ingestão de álcool em grandes quantidades, o diabetes mellitus, o tabagismo, o sedentarismo, o estresse, a obesidade e o colesterol elevado ${ }^{3}$. Já entre os fatores não modificáveis, destacase a idade, havendo clara relação entre o envelhecimento e o risco de desenvolver DCNTs. Outros fatores não modificáveis são a hereditariedade, o sexo e a raça.

O envelhecimento da população e a heterogeneidade demográfica, social e econômica observada no Brasil se refletem em diferentes padrôes de mortalidade e de morbidade por DCNTs, exigindo respostas que envolvam as especificidades locais e que sejam adequadas às suas realidades ${ }^{1}$. $\mathrm{O}$ conhecimento da prevalência dos fatores de risco para DCNTs, principalmente os de natureza comportamental, isto é, os que podem ser modificados, é fundamental por serem sinais de alerta para o crescimento da morbimortalidade relacionada a essas doenças ${ }^{1}$. Os inquéritos de saúde de base populacional, principalmente os de alcance nacional, constituem o principal instrumento utilizado para conhecer a prevalência dos fatores de risco para doenças crônicas ${ }^{2}$. No Brasil, entretanto, a literatura na área de saúde pública ainda é escassa em textos sobre prevalência dos fatores de risco para as $\mathrm{DCNTs}^{4}$. O que há disponível, na maioria das vezes, são informações para algumas cidades, não podendo-se comparar os resultados devido às diferenças nos instrumentos de coleta utilizados. Este estudo teve por objetivo conhecer a prevalência da exposição da população aos principais fatores de risco para o desenvolvimento de DCNTs no Brasil.

\section{METODOLOGIA}

Uma revisão sistemática da literatura foi realizada no mês de novembro de 2008 a partir das bases de dados on-line Lilacs (Literatura Latino-Americana e do Caribe em Ciências da Saúde) e Medline (Literatura Internacional em Ciências da Saúde), no período de 2003 a 2008. Foram utilizadas as seguintes palavraschave (em português e inglês): prevalência de fatores de risco, doença crônica não transmissível e Brasil. Essas bases foram acessadas por meio da Biblioteca Virtual em Saúde Pública da Bireme/OPAS/OMS (http:// www.bvs.br). A revisão foi ampliada por meio da busca a referências bibliográficas dos estudos relevantes, solicitação de estudos publicados a especialistas e busca em outras fontes. Não houve limite com relação à idade das populações estudadas.

Foram selecionados somente artigos publicados em periódicos científicos e que preenchiam os seguintes critérios: ser um estudo transversal, ter sido realizado no Brasil, conter informaçōes sobre a prevalência de pelo menos um dos seguintes fatores de risco para as DCNTs: alimentação, atividade física, consumo de cigarros e de bebidas alcoólicas, obesidade e autorreferência a diagnóstico médico de hipertensão arterial e diabetes mellitus, entre outros quesitos. Foram excluídos os artigos sem resumo e as duplicidades. Um total de 12 artigos foi selecionado atendendo a esses critérios.

\section{RESULTADOS}

Os dados descritivos dos métodos utilizados nos estudos transversais sobre fatores de risco para DCNTs publicados no Brasil, entre 2003-2008, como: nome dos autores; localidade; ano de realização do estudo; tipo de amostragem; número de participantes; faixa etária; material de cada estudo; isto é, se o inquérito foi domiciliar ou telefônico; e a qual a bibliografia de base para elaboração do instrumento usado na coleta de dados, são apresentados na tabela $1^{5-16}$. Observa-se que apenas dois estudos ${ }^{8,10}$ não fazem referência aos seus instrumentos, descrevendo apenas as variáveis pesquisadas. É importante observar que há diferenças metodológicas entre os estudos com relação aos seguintes 
aspectos: representatividade da amostra e faixa etária da população entrevistada. Dos 12 estudos analisados, seis foram realizados por meio telefônico e utilizaram delineamento transversal de base populacional, baseandose em amostra probabilística da população adulta residente em domicílios servidos por linhas fixas de telefone.

No Quadro 1, é apresentada uma síntese dos estudos que analisaram a presença de um ou mais fatores de risco para DCNTs no território nacional. Com relação à análise das características sociodemográficas, as variáveis sexo e idade estiveram presentes em 100\% dos estudos e a variável escolaridade, em 90,9\%. O índice de massa corporal (IMC) - obesidade, excesso de peso - e dislipidemia apareceram como os fatores de risco mais investigados, seguidos do tabagismo, hipertensão arterial referida, diabetes mellitus, atividade física, consumo excessivo de álcool e consumo de frutas, verduras e hortaliças.

Tabela 1. Dados descritivos dos métodos utilizados nos estudos transversais sobre fatores de risco para DCNTs, Brasil, 2003-2008

\begin{tabular}{|c|c|c|c|c|c|c|c|}
\hline $\begin{array}{c}\text { Autor } \\
\text { (ano da } \\
\text { publicação) }\end{array}$ & Localidade & $\begin{array}{l}\text { Ano de } \\
\text { realização }\end{array}$ & $\begin{array}{l}\text { Tipo de } \\
\text { amostra }\end{array}$ & $\begin{array}{l}\text { Número de } \\
\text { participantes }\end{array}$ & Faixa etária & $\begin{array}{l}\text { Tipo de } \\
\text { inquérito }\end{array}$ & Questionário utilizado \\
\hline $\begin{array}{l}\text { Dias da Costa ef } \\
\text { al. }(2004)^{5}\end{array}$ & Pelotas - RS & $1999-2000$ & $\begin{array}{l}\text { Múltiplos } \\
\text { estágios }\end{array}$ & 2.177 & 20 a 69 anos & Domiciliar & $\begin{array}{l}\text { Específico para o estudo, } \\
\text { baseado nos } \\
\text { questionários de Moreira } \\
\text { et al. (1996) e do } \\
\text { National Institute of } \\
\text { Health (1997) }\end{array}$ \\
\hline $\begin{array}{l}\text { Souza et al. } \\
(2003)^{6}\end{array}$ & $\begin{array}{l}\text { Campos dos } \\
\text { Goytacazes - RJ }\end{array}$ & 2001 & Conglomerados & 1.039 & 18 anos ou + & Domiciliar & Específico para o estudo \\
\hline $\begin{array}{l}\text { Lessa } \\
(2004)^{7}\end{array}$ & Salvador-BA & 2003 & Conglomerados & 1.439 & 20 anos ou + & Domiciliar & $\begin{array}{l}\text { Específico do Projeto de } \\
\text { Monitoramento das } \\
\text { Doenças Cardiovasculares } \\
\text { e do Diabetes no Brasil } \\
\text { (MONIT) }\end{array}$ \\
\hline $\begin{array}{l}\text { Marcopito et al. } \\
(2005)^{8}\end{array}$ & São Paulo - SP & $2001-2002$ & Probabilística & 1.968 & 15 a 59 anos & Domiciliar & Específico para o estudo \\
\hline $\begin{array}{l}\text { Monteiro et al. } \\
(2005)^{9}\end{array}$ & São Paulo - SP & 2003 & Probabilística & 2.122 & 18 anos ou + & Telefônico & $\begin{array}{l}\text { SIMTEL/ MSP específico, } \\
\text { baseado nos } \\
\text { questionários de } \\
\text { Remington ef al. (1988) e } \\
\text { da OMS (2001) }\end{array}$ \\
\hline $\begin{array}{l}\text { Yokota et al. } \\
(2007)^{10}\end{array}$ & $\begin{array}{l}\text { Sobradinho e São } \\
\text { Sebastião - DF }\end{array}$ & $2005-2006$ & Aleatória & 157 & 18 anos ou + & Domiciliar & $\begin{array}{l}\text { Questionário específico } \\
\text { para o estudo }\end{array}$ \\
\hline $\begin{array}{l}\text { Carvalhaes et al. } \\
(2008)^{11}\end{array}$ & Botucatu - SP & 2004 & Probabilística & 1.410 & 18 anos ou + & Telefônico & SIMTEL/ Botucatu \\
\hline $\begin{array}{l}\text { Moura ef al. } \\
(2008)^{12}\end{array}$ & 26 Estados e DF & 2006 & Probabilística & 54.369 & 18 anos ou + & Telefônico & $\begin{array}{l}\text { Questionário específico, } \\
\text { baseado nos } \\
\text { questionários de } \\
\text { Remington et al. (1988), } \\
\text { da OMS (2001) e do } \\
\text { SIMTEL (2005) }\end{array}$ \\
\hline $\begin{array}{l}\text { Figueiredo et al. } \\
(2008)^{13}\end{array}$ & São Paulo - SP & 2003 & Probabilística & 2.122 & 18 anos ou + & Telefônico & SIMTEL/MSP \\
\hline $\begin{array}{l}\text { Peixoto ef al. } \\
(2008)^{14}\end{array}$ & Goiânia - G0 & 2005 & Probabilística & 2.002 & 18 anos ou + & Telefônico & SIMTEL/ Goiânia \\
\hline $\begin{array}{l}\text { Cunha et al. } \\
(2008)^{15}\end{array}$ & Goiânia-G0 & 2005 & Probabilística & 2.002 & 18 anos ou + & Telefônico & SIMTEL/ Goiânia \\
\hline $\begin{array}{l}\text { Pereira et al. } \\
(2008)^{16}\end{array}$ & $\begin{array}{l}15 \text { capitais } \\
\text { brasileiras e DF }\end{array}$ & $2002 / 2003$ & Probabilística & 3.142 & $\begin{array}{l}60 \text { anos ou }+ \\
\text { (idosos) }\end{array}$ & Domiciliar & $\begin{array}{l}\text { Questionário específico } \\
\text { para o estudo }\end{array}$ \\
\hline
\end{tabular}


Quadro 1. Principais fatores de risco investigados nos estudos transversais selecionados, Brasil 2003-2008

\begin{tabular}{|c|}
\hline Fatores estudados \\
\hline $\operatorname{Sexo}^{5,6,7,8,9,10,11,12,13,14,15,16}$ \\
\hline Idade $5,6,7,8,9,10,11,12,13,14,15,16$ \\
\hline Cor da pele/ Raça ${ }^{5,6,7}$ \\
\hline Escolaridade $5,6,7,9,10,11,12,13,14,15,16$ \\
\hline $\begin{array}{l}\text { Classe social, nível social (ANEP), nível } \\
\text { socioeconômico } 5,6,7\end{array}$ \\
\hline Estado civil ${ }^{5,6,9,15}$ \\
\hline Etnicidade $^{12}$ \\
\hline Tabagismo $^{5,7,8,9,10,11,12,14,16}$ \\
\hline Consumo de álcool $5,7,9,11,12,14,16$ \\
\hline $\begin{array}{l}\text { Índice de massa corporal (obesidade, excesso de } \\
\text { peso) } 5,7,8,9,10,11,12,14,15,16\end{array}$ \\
\hline Circunferência da cintura ${ }^{7}$ \\
\hline Circunferência do abdômen ${ }^{8,10}$ \\
\hline Circunferência do quadril ${ }^{8}$ \\
\hline Atividade física (sedentarismo) $5,9,10,11,12,14,15,16$ \\
\hline $\begin{array}{l}\text { Consumo de frutas, verduras e } \\
\text { hortaliças } 9,10,11,12,13,14,16\end{array}$ \\
\hline Hipertensão arterial referida $a^{5,7,9,11,12,14,15,16}$ \\
\hline Pressão arterial medida $5,6,7,8,10,16$ \\
\hline Diabetes mellitus referida $6,7,9,11,12,14,15,16$ \\
\hline Dislipidemia $^{6,7,8,9,10,12,14,15,16}$ \\
\hline Doença crônica referida $5,7,12,16$ \\
\hline Avaliação do estado de saúde 9,12 \\
\hline
\end{tabular}

As Tabelas 2A e 2B comparam as prevalências dos diferentes fatores encontrados nos estudos selecionados. A prevalência de fumantes variou de $8,7 \%{ }^{14}$ a $19,3 \%{ }^{11}$ entre as mulheres e entre os homens de $15,1 \%{ }^{14}$ a $24,8 \%{ }^{11}$. Já a prevalência de consumo excessivo de álcool em mulheres esteve entre $0,1 \%^{11}$ e $14,4 \%^{14}$ e nos homens entre $3,5 \%{ }^{7}$ a $37,7 \%{ }^{14}$. Por outro lado, a prevalência de excesso de peso entre as mulheres oscilou de $1,5 \%{ }^{7}$ a $52,0 \%{ }^{10}$ e entre os homens de $7,7 \%{ }^{7}$ a $47,0 \%{ }^{10,12}$. Além disso, a prevalência de sedentarismo em mulheres esteve entre $20,1 \%{ }^{12}$ a $58,2 \%{ }^{11}$ e nos homens entre $33,9 \%{ }^{9}$ e $43,1 \%{ }^{11}$. A prevalência de hipertensão arterial variou de $5,3 \%{ }^{7}$ a $30,0 \%{ }^{10}$ entre as mulheres e entre os homens de $10,9 \%{ }^{15}$ a $34,0 \%{ }^{10}$.

\section{DISCUSSÃO}

Atualmente as DCNTs representam uma grande preocupação na área da Saúde Pública. Para que seja possível monitorar os comportamentos de risco que levam a população a desenvolver DCNTs é preciso que se tenha o conhecimento do perfil de exposição populacional aos seus fatores de risco, ou seja, do seu estilo de vida. O presente estudo dedicou-se à revisão sistemática da literatura científica sobre estudos transversais populacionais realizados no Brasil que apresentavam a prevalência dos fatores de risco para as DCNTs. Os artigos encontrados referiam-se à avaliação de algum dos seguintes fatores de risco: consumo abusivo de álcool; ser tabagista; sobrepeso; excesso de peso e obesidade (definidos a partir do IMC); circunferência da cintura; inatividade física; frequência do consumo de frutas, legumes e verduras; ter diabetes mellitus; hipertensão arterial; e dislipidemia. Entretanto nota-se que, embora alguns estudos tenham descrito no item "questionário" a presença da variável que avalia dislipidemia, esta informação não aparece nos resultados publicados.

Observou-se que os estudos transversais brasileiros sobre fatores de risco para DCNTs são recentes e em pequeno número, com resultados, pelo menos, para todas as capitais brasileiras e o Distrito Federal (DF). A maioria dos estudos concentra-se na região Sudeste do País. Apenas um estudo apresentou resultados para as capitais dos 26 Estados brasileiros e o $\mathrm{DF}^{12}$, mesmo assim com algumas limitaçôes por ser um inquérito telefônico. Estudos individuais de base populacional sobre prevalência dos fatores de risco para DCNTs já foram realizados em cidades tão populosas como São Paulo e de menor porte como Campos dos Goytacazes, no Estado do Rio de Janeiro. Como esses dados estão publicados de modo não uniforme, isso dificulta comparações entre os estudos.

A prevalência de tabagismo para o Brasil, $16,2 \%{ }^{12}$, caiu se comparada com aquela observada na Pesquisa Nacional de Saúde e Nutrição realizada em 1989 pelo Instituto Brasileiro de Geografia Estatística (IBGE) ${ }^{24}$ : $30,2 \%$. Embora as metodologias desses estudos não sejam comparáveis, os resultados apontam para uma expressiva redução nos percentuais.

O consumo abusivo de álcool ocasiona consequências negativas para a saúde e a qualidade de vida dos indivíduos $(0,1 \%$ a $37,7 \%)$. Com relação à prevalência desse comportamento, há grandes diferenças entre as cidades. Isso se deve ao fato de não ter sido usado um questionário padronizado para esse importante fator de risco.

No que se refere à prevalência de excesso de peso, os resultados ficaram entre $40 \%$ e $50 \%$, na maioria dos estudos. Estes dados se aproximam pelo fato de haver um consenso no que diz respeito à padronização do cálculo do IMC.

Hipertensão arterial também apresentou diferenças importantes $(5,3 \%$ a $34,0 \%)$, mas explicáveis pelo ponto 
Tabela 2A. Prevalência dos fatores de risco para DCNTs encontrados nos estudos transversais selecionados, Brasil 2003-2008

\begin{tabular}{|c|c|c|c|c|c|}
\hline \multirow{2}{*}{$\begin{array}{c}\text { Autor } \\
\text { Ano da } \\
\text { publicação }\end{array}$} & \multicolumn{5}{|c|}{ Fatores de Risco (\%) } \\
\hline & Tabagismo & $\begin{array}{c}\text { Consumo abusivo de } \\
\text { álcool }\end{array}$ & IMC & Inatividade física & $\begin{array}{c}\text { Consumo de frutas, legumes } \\
\text { e verduras }\end{array}$ \\
\hline $\begin{array}{l}\text { Dias da Costa et } \\
\text { al. }(2004)^{5}\end{array}$ & $\begin{array}{l}\text { Leve/moderado: } \\
\mathrm{T}=16,7 \\
\text { Pesado: } \\
\mathrm{T}=28,8\end{array}$ & $\begin{array}{l}T=14,3 ; H=29,2 ; \\
M=3,7\end{array}$ & $\begin{array}{l}\text { Sobrepeso: } \\
\mathrm{T}=33,7 \\
\text { Obesidade: } \\
\mathrm{T}=19,4\end{array}$ & $\begin{array}{l}\text { Insuficientemente ativo: } \\
T=80,6\end{array}$ & --- \\
\hline $\begin{array}{l}\text { Souza ef al. } \\
\qquad(2003)^{6}\end{array}$ & --- & --- & $\begin{array}{l}\text { Excesso de peso (IMC }>25): \\
T=10,6\end{array}$ & --- & --- \\
\hline $\begin{array}{l}\text { Lessa } \\
(2004)^{7}\end{array}$ & --- & $H=3,5 ; M=7,7$ & $\begin{array}{l}\text { Excesso de peso }(I M C \geq 25) \text { : } \\
H=7,7 ; M=1,5 \\
\text { Circunferência abdominal } \\
\text { aumentada: } \\
H=6,4 ; M=4,2\end{array}$ & --- & --- \\
\hline $\begin{array}{l}\text { Marcopito et al. } \\
\qquad(2005)^{8}\end{array}$ & $\mathrm{~T}=22,6$ & --- & $\begin{array}{l}\text { Obesidade: } \\
T=13,7 \\
\text { Circunferência abdominal } \\
\text { aumentada: } \\
T=19,7\end{array}$ & --- & --- \\
\hline $\begin{array}{l}\text { Monteiro et al. } \\
(2005)^{9}\end{array}$ & $\begin{array}{l}T=20,8 ; H=23,8 \\
M=18,2\end{array}$ & $\begin{array}{l}2 \text { doses diárias pelo } \\
\text { menos } 1 \text { vez por } \\
\text { semana: } \\
T=15,0 ; H=23,9 ; \\
M=7,2 \\
2 \text { doses todo dia ou } \\
\text { quase todo dia: } \\
T=3,8 ; H=7,4 ; \\
M=0,6\end{array}$ & $\begin{array}{l}\text { Excesso de peso: } \\
T=40,8 ; H=45,4 ; M=36,3 \\
\text { Obesidade: } \\
T=10,0 ; H=9,4 ; M=10,5\end{array}$ & $\begin{array}{l}\text { Sedentarismo: } \\
T=46,7 ; H=33,9 ; \\
M=57,9\end{array}$ & $\begin{array}{l}\text { Frutas: } 5 \text { ou mais dias na } \\
\text { semana: } \\
T=48,9 ; H=39,5 ; \\
M=57,1 \\
\text { Hortaliças cruas: } 5 \text { ou mais } \\
\text { dias na semana: } \\
T=56,8 ; H=52,3 ; \\
M=60,7 \\
\text { Hortaliças cozidas: } 5 \text { ou } \\
\text { mais dias na semana: } \\
T=24,9 ; H=17,6 ; \\
M=31,2\end{array}$ \\
\hline $\begin{array}{l}\text { Yokota ef al. } \\
\qquad(2007)^{10}\end{array}$ & $\begin{array}{l}T=18,0 ; H=22,0 ; \\
M=16,0\end{array}$ & -- & $\begin{array}{l}\text { Excesso de peso: } \\
T=49,0 ; H=47,0 ; M=52,0 \\
\text { Circunferência abdominal } \\
\text { aumentada: } \\
T=31,0 ; H=19,0 ; M=39,0\end{array}$ & --- & $\begin{array}{l}\text { Baixo consumo diário de } \\
\text { frutas: } \\
T=69,0 ; H=73,0 ; \\
M=67,0 \\
\text { Baixo consumo diário de } \\
\text { hortaliças: } \\
T=52,0 ; H=62,0 ; \\
M=45,0\end{array}$ \\
\hline $\begin{array}{l}\text { Carvalhaes ef al. } \\
\qquad(2008)^{11}\end{array}$ & $\begin{array}{l}T=22,0 ; H=24,8 \\
M=19,3\end{array}$ & $\begin{array}{l}1 \text { dia: } \\
T=25,2 ; H=32,8 ; \\
M=11,7 \\
5 \text { dias: } \\
T=3,2 ; H=6,5 ; \\
M=0,1\end{array}$ & $\begin{array}{l}\text { Excesso de peso: } \\
T=46,7 ; H=48,3 ; M=45,1 \\
\text { Obesidade: } \\
T=15,7 ; H=13,8 ; M=17,6\end{array}$ & $\begin{array}{l}\text { Sedentarismo: } \\
T=57,9 ; H=43,1 ; \\
M=58,2\end{array}$ & $\begin{array}{l}\text { Frutas: } \\
T=50,5 ; H=48,6 ; \\
M=52,3 \\
\text { Hortaliças cruas: } \\
T=79,1 ; H=76,6 ; \\
M=81,4 \\
\text { Hortaliças cozidas: } \\
T=59,4 ; H=56,8 ; \\
M=61,8\end{array}$ \\
\hline $\begin{array}{l}\text { Moura ef al. } \\
(2008)^{12}\end{array}$ & $\begin{array}{l}T=16,2 ; H= \\
20,3 ; M=12,8\end{array}$ & $\begin{array}{l}T=16,1 ; H=25,3 ; \\
M=8,1\end{array}$ & $\begin{array}{l}\text { Excesso de peso: } \\
T=43,0 ; H=47,3 ; M=38,8\end{array}$ & $\begin{array}{l}\text { Sedentarismo: } \\
T=29,2 ; H=39,8 ; \\
M=20,1\end{array}$ & $\begin{array}{l}T=23,9 ; H=17,8 ; \\
M=29,1\end{array}$ \\
\hline
\end{tabular}

$\mathrm{T}=$ Total; H=Homens; M=Mulheres 
Tabela 2A. (cont.)

\begin{tabular}{|c|c|c|c|c|c|}
\hline \multirow{2}{*}{$\begin{array}{c}\text { Autor } \\
\text { Ano da } \\
\text { publicação }\end{array}$} & \multicolumn{5}{|c|}{ Fatores de Risco (\%) } \\
\hline & Tabagismo & $\begin{array}{c}\text { Consumo abusivo de } \\
\text { álcool }\end{array}$ & IMC & Inatividade física & $\begin{array}{c}\text { Consumo de frutas, legumes } \\
\text { e verduras }\end{array}$ \\
\hline $\begin{array}{l}\text { Figueiredo ef al. } \\
(2008)^{13}\end{array}$ & --- & --- & --- & --- & $\begin{array}{l}\text { Consumo diário de frutas: } \\
T=43,9 ; H=35,0 ; \\
M=51,7 \\
\text { Consumo diário de } \\
\text { verduras: } \\
T=46,8 ; H=39,9 ; \\
M=52,7 \\
\text { Consumo diário de } \\
\text { legumes: } \\
T=18,1 ; H=11,4 ; \\
M=23,9\end{array}$ \\
\hline $\begin{array}{l}\text { Peixoto et al. } \\
(2008)^{14}\end{array}$ & $\begin{array}{l}T=11,1 ; H=15,1 ; \\
M=8,7\end{array}$ & $\begin{array}{l}T=23,2 ; H=37,7 ; \\
M=14,4\end{array}$ & $\begin{array}{l}\text { Excesso de peso: } \\
T=36,5 ; H=45,3 ; M=30,9 \\
\text { Obesidade: } \\
T=10,6 ; H=11,0 ; M=10,3\end{array}$ & $\begin{array}{l}\text { Sedentarismo: } \\
T=29,7 ; H=34,8 ; \\
M=26,6\end{array}$ & $\begin{array}{l}\text { Consumo regular de frutas: } \\
T=51,5 ; H=43,5 ; \\
M=56,3 \\
\text { Consumo regular de } \\
\text { hortaliças: } \\
T=81,6 ; H=76,4 ; \\
M=84,8\end{array}$ \\
\hline $\begin{array}{l}\text { Cunha et al. } \\
(2008)^{15}\end{array}$ & --- & --- & $\begin{array}{l}\text { Obesidade em ativos: } \\
H=7,8 ; M=7,3 \\
\text { Obesidade em Inativos: } \\
H=11,5 ; M=10,2 \\
\text { Excesso de peso em ativos: } \\
H=37,8 ; M=25,0 \\
\text { Excesso de peso em inativos: } \\
H=46,3 ; M=32,5\end{array}$ & $H=42,0 ; M=55,5$ & --- \\
\hline $\begin{array}{l}\text { Pereira et al. } \\
(2008)^{16}\end{array}$ & $\begin{array}{l}T=12,7 H=14,6 ; \\
M=6,0\end{array}$ & $\begin{array}{l}T=3,2 H=5,2 ; \\
M=1,2\end{array}$ & $\begin{array}{l}\text { Obesidade: } \\
H=12,8 ; M=19,4\end{array}$ & $H=41,8 ; M=35,7$ & $\begin{array}{l}<5 \text { porções por dia } \\
H=96,5 ; M=93,4\end{array}$ \\
\hline
\end{tabular}

$\mathrm{T}=$ Total; $\mathrm{H}=$ Homens; $\mathrm{M}=$ Mulheres

de corte que mudou nos últimos anos, de acordo com a Classificação de Pressão Arterial de 2004 da Sociedade Brasileira de Hipertensão ${ }^{25}$. Esse fato influenciou a baixa prevalência em algumas cidades, em função do ano de coleta de dados. Isto foi observado no resultado de Souza et al. ${ }^{6}$, que se baseou nos critérios definidos pelo VI Joint National Committee, de 1997.

A prevalência de sedentarismo também variou muito entre os estudos, mas o resultado para Brasil, 29,2\% ${ }^{12}$, apesar de ainda preocupar, aponta um decréscimo se comparado com os outros estudos isolados e a valores observados em diferentes países. Isso se deve ao indicador gerado em cada estudo: atividade física de lazer, dias por semana com atividade física no lazer (pelo menos 30 minutos $)^{11,15}$, atividade física ocupacional ${ }^{15}$, atividade física no deslocamento ${ }^{11,15}$, atividade física na limpeza ${ }^{15}$, fisicamente inativos (sem prática de qualquer atividade física no lazer nos últimos três meses e que não realizam esforços físicos intensos no trabalho, não se deslocam para o trabalho caminhando ou de bicicleta e não são responsáveis pela limpeza pesada de suas casas) ${ }^{11}$ e sedentarismo ${ }^{11,15}$.

O consumo de frutas, verduras e hortaliças apresentou diferenças relevantes entre as localidades analisadas. Entretanto é preciso entender como foi construído o indicador que variou muito na sua forma de apresentação: consumo regular ${ }^{14}$, consumo diário ${ }^{13}$, consumo em cinco ou mais dias na semana ${ }^{9,11}$, baixo consumo diário ${ }^{10}$, ingestão inferior a cinco porçôes por dia $^{16}$.

Por sua vez, diabetes mellitus e dislipidemia também apresentaram resultados que podem ter sido influenciados pelo ponto de corte definido pelas Diretrizes Brasileiras sobre Dislipidemias e Diretriz de Prevenção de Aterosclerose do Departamento de Aterosclerose da Sociedade Brasileira de Cardiologia ${ }^{17}$.

Nessa revisão ficam claras ainda as diferenças com relação aos instrumentos de coleta utilizados, o que prejudica a comparação entre as localidades. Foi observado 
Tabela 2B. Prevalência dos fatores de risco para DCNTs encontrados nos estudos transversais selecionados, Brasil 2003-2008

\begin{tabular}{|c|c|c|c|}
\hline \multirow{2}{*}{$\begin{array}{c}\text { Autor } \\
\text { Ano da publicação }\end{array}$} & \multicolumn{3}{|c|}{ Fatores de Risco (\%) } \\
\hline & Hipertensão arterial & Diabetes Mellitus & Dislipidemia \\
\hline $\begin{array}{l}\text { Dias da Costa et al. } \\
(2004)^{5}\end{array}$ & $\mathrm{~T}=23,5$ & $\cdots$ & -- \\
\hline $\begin{array}{l}\text { Souza et al. } \\
(2003)^{6}\end{array}$ & $\mathrm{~T}=13,4$ & $\mathrm{~T}=6,2$ & $\begin{array}{l}\text { Colesterol Total } \geq 240 \mathrm{mg} / \mathrm{dL}=11,0 \\
\mathrm{HDL}-\mathrm{c} \leq 40 \mathrm{mg} / \mathrm{dL}=13,2 \\
\mathrm{LDL}-\mathrm{c} \geq 160 \mathrm{mg} / \mathrm{dL}=8,4 \\
\text { Triglicerídeos } \geq 200 \mathrm{mg} / \mathrm{dL}=14,9\end{array}$ \\
\hline $\begin{array}{l}\text { Lessa } \\
(2004)^{7}\end{array}$ & $H=11,5 ; M=5,3$ & $H=6,3 ; M=2,7$ & $\begin{array}{l}\text { Colesterol: } H=6,1 ; M=2,8 \\
\text { HDL: } H=4,6 ; M=1,8 ; \\
\text { LDL: } H=5,8 ; M=2,4 \\
\text { Triglicérides: } H=4,2 ; M=1,0\end{array}$ \\
\hline $\begin{array}{l}\text { Marcopito et al. } \\
(2005)^{8}\end{array}$ & $\mathrm{~T}=24,3$ & Glicemia $\geq 110 \mathrm{mg} / \mathrm{dl}=6,8$ & $\begin{array}{l}\text { Colesterol total } \geq 240 \mathrm{mg} / \mathrm{dl}=8,1 \\
\mathrm{HDL} \text {-colesterol }<40 \mathrm{mg} / \mathrm{dl}=27,1 \\
\text { Triglicérides } \geq 200 \mathrm{mg} / \mathrm{dl}=14,4\end{array}$ \\
\hline $\begin{array}{l}\text { Monteiro et al. } \\
(2005)^{9}\end{array}$ & $\mathrm{~T}=21,9 ; \mathrm{H}=18,2 ; \mathrm{M}=25,0$ & $\mathrm{~T}=5,4 ; \mathrm{H}=5,0 ; M=5,8$ & -- \\
\hline $\begin{array}{l}\text { Yokota et al. } \\
(2007)^{10}\end{array}$ & $\mathrm{~T}=31,0 ; \mathrm{H}=34,0 ; \mathrm{M}=30,0$ & --- & --- \\
\hline $\begin{array}{l}\text { Carvalhaes et al. } \\
(2008)^{11}\end{array}$ & $\mathrm{~T}=23,1 ; \mathrm{H}=21,4 ; \mathrm{M}=24,6$ & $\mathrm{~T}=5,9 ; \mathrm{H}=3,8 ; \mathrm{M}=7,8$ & -- \\
\hline $\begin{array}{l}\text { Moura ef al. } \\
(2008)^{12}\end{array}$ & --- & -- & -- \\
\hline $\begin{array}{l}\text { Figueiredo ef al. } \\
(2008)^{13}\end{array}$ & -- & -- & --- \\
\hline $\begin{array}{l}\text { Peixoto et al. } \\
(2008)^{14}\end{array}$ & $\mathrm{~T}=22,4 ; \mathrm{H}=20,0 ; \mathrm{M}=23,8$ & $\mathrm{~T}=4,4 ; \mathrm{H}=4,3 ; \mathrm{M}=4,4$ & $\mathrm{~T}=18,4 ; \mathrm{H}=14,1 ; \mathrm{M}=21,0$ \\
\hline $\begin{array}{l}\text { Cunha et al. } \\
(2008)^{15}\end{array}$ & $\begin{array}{l}\text { Ativos: } H=10,9 ; M=20,1 \\
\text { Inativos: } H=20,0 ; M=21,6\end{array}$ & $\begin{array}{l}\text { Ativos: } H=1,8 ; M=2,6 \\
\text { Inativos: } H=4,6 ; M=4,4\end{array}$ & $\begin{array}{l}\text { Ativos: } H=9,0 ; M=22,8 \\
\text { Inativos: } H=12,8 ; M=15,5\end{array}$ \\
\hline $\begin{array}{l}\text { Pereira ef al. } \\
(2008)^{16}\end{array}$ & $\mathrm{~T}=50,6 ; H=43,5 ; M=52,4$ & $T=17,8 ; H=21,6 ; M=14,9$ & $\mathrm{~T}=33,1 ; \mathrm{H}=23,5 ; \mathrm{M}=32,5$ \\
\hline
\end{tabular}

$\mathrm{T}=$ Total; $\mathrm{H}=$ Homens; $\mathrm{M}=$ Mulheres

que alguns autores relatam a fonte dos seus questionários, ou seja, a base bibliográfica usada para a construção do instrumento utilizado para a coleta dos dados, enquanto outros apenas descrevem as variáveis usadas.

Por outro lado, a principal limitação das entrevistas telefônicas diz respeito à exclusão de indivíduos que moram em domicílios sem telefone fixo. No Brasil, apesar do crescimento da rede de telefonia fixa nos últimos anos, sabe-se que ainda não é universal e igualitária, podendo ser particularmente baixa em cidades economicamente menos desenvolvidas e nos Estados com menor índice socioeconômico. Segundo dados da Pesquisa de Orçamentos Familiares (POF), realizada pelo IBGE, entre 2002 e 2003, 66,4\% do conjunto dos domicílios das 27 capitais declararam despesa com serviço de telefonia fixa, variando de
33,7\% em Macapá a 79,4\% no DF. O Vigitel, apesar dessas limitaçōes, vem estabelecendo uma série temporal de medidas de prevalência que têm permitido a padronização e a comparabilidade dos dados.

\section{CONCLUSÃO}

Conclui-se que a variação nos percentuais de exposição populacional aos fatores de risco para as DCNTs presentes nos diversos grupos populacionais aponta para a necessidade de padronização dos instrumentos de coleta de dados para que se possam comparar as prevalências encontradas nos diferentes inquéritos (comparação entre localidades) e em diferentes ocasiōes (comparação temporal). Além disso, as altas prevalências obtidas nos estudos realizados indicam a 
necessidade de intervenções, visando à implementação de estratégias de prevenção e promoção da saúde para melhorar o perfil dos brasileiros no que diz respeito à exposição aos fatores de risco para as DCNTs.

\section{Declaração de Conflito de Interesses: Nada a Declarar.}

1. Brasil. Ministério da Saúde. A vigilância, o controle e a prevenção das doenças crônicas não transmissíveis: DCNT no contexto do Sistema Único de Saúde Brasileiro. Epidemiologia e serviços de saúde : revista do Sistema Único de Saúde do Brasil 2006; 15(1) : 47 - 65.

2. Barros MBA, César CLG, Carandina L, Torre GD. Desigualdades sociais na prevalência de doenças crônicas no Brasil, PNAD-2003. Cien Saude Colet 2006; 11(4): 911-26.

3. Botrel TEA, Costa RD, Costa MD, Costa AMD. Doenças cardiovasculares: causas e prevenção / Cardiovascular diseases: etiology and prevention, Revista brasileira de clínica e terapêutica 2000 maio; 26(3):87-90.

4. Lessa I. Doenças crônicas não-transmissíveis no Brasil: um desafio para a complexa tarefa de vigilância. Cien Saude Colet 2004; 9(4): 931-43.

5. Costa JD, Silveira MF, Gazalle FK, Oliveira SS, Hallal PC, Menezes ANB, Gigante DP, Olinto MTA, Macedo S. Consumo abusivo de álcool e fatores associados: estudo de base populacional. Rev Saude Publica 2004; 38 (2): 284-91.

6. Souza LJ, Chalita FEB, Reis AFF, Texeira CL, Gicovate Neto C, Bastos DA, S Filho JTD, Souza TF, Côrtes VA. Prevalência de Diabetes Mellitus e fatores de risco em Campos dos Goytacazes, RJ. Arq Bras Endocrinol Metabol $2003 \mathrm{fev}$; 47(1): 69-74.

7. Lessa I. Níveis séricos de creatinina: hipercreatininemia em segmento da população adulta de Salvador, Brasil. Revista brasileira de epidemiologia 2004; 7( 2): 176-186.

8. Marcopito LF, Rodrigues SS, Pacheco MA, Shirassu MM, Goldfeder AJ, Moraes MA. Prevalence of a set of risk factors for chronic diseases in the city of São Paulo, Brazil. Rev Saude Publica 2005 Oct; 39(5): 738-45.

9. Monteiro CA, Moura EC, Jaime PC, Lucca A, Florindo AA, Figueiredo ICR, Bernal R, Silva NN. Monitoramento de fatores de risco para doenças crônicas por entrevistas telefônicas. Rev Saude Publica 2005; 39 (1): 47-57.

10. Yokota, RTC, Vasconcelos TF, Ito MK, Dutra ES, Baiocchi KC, Hamann EM, Lopes EB, Barboza RB. Prevalência de fatores de risco para doenças crônicas não-transmissíveis em duas regiôes do Distrito Federal. Comunicação em Ciencias da Saúde 2007; 18(4): 289-96.

11. Carvalhaes MABL, Moura EC, Monteiro CA. Prevalência de fatores de risco para doenças crônicas: inquérito populacional mediante entrevistas telefônicas em Botucatu, São Paulo, 2004. Revista brasileira de epidemiologia 2008; 11(1): 14-23.
12. Moura EC, Monteiro CA, Claro RM, Bernal R, Silva NN, Moura L, Malta DC, Moraes Neto OL. Vigilância de Fatores de Risco para Doenças Crônicas por Inquérito Telefônico nas capitais dos 26 estados brasileiros e no Distrito Federal (2006). Revista brasileira de epidemiologia 2008; 11 (supl 1): 20-37.

13. Figueiredo ICR, Jaime PC, Monteiro CA. Factors associated with fruit and vegetable intake among adults of the city of São Paulo, Southeastern Brazil. Rev Saude Publica 2008;42(5):777-85.

14. Peixoto MRG, Monego ET, Alexandre VP, Souza RGM, Moura EC. Monitoramento por entrevistas telefônicas de fatores de risco para doenças crônicas: experiência de Goiânia, Goiás, Brasil. Cad Saude Publica 2008 jun; 24(6):1323-33.

15. Cunha IC, Peixoto MRG, Jardim PCBV, Alexandre VP. Fatores associados à prática de atividade física na população adulta de Goiânia: monitoramento por meio de entrevistas telefônicas. Revista brasileira de epidemiologia 2008; 11(3): 495-504.

16. Pereira JC, Barreto SM, Passos VM. O perfil de saúde cardiovascular dos idosos brasileiros precisa melhorar: estudo de base populacional. Arq Bras Cardiol 2008; 91 (1); 1-10.

17. Sociedade Brasileira de Cardiologia. III Diretrizes Brasileiras Sobre Dislipidemias e Diretriz de Prevenção da Aterosclerose do Departamento de Aterosclerose da Sociedade Brasileira de Cardiologia. [acesso em: 2008 nov 26]. Disponível em: http://bvsms.saude.gov.br/bvs/publicacoes/ diretrizcorreta.pdf.

18. Berrigan D, Dodd K., Troiano RP, Krebs-Smith SM, Barbash RB. Patterns of health behavior in U.S. adults. Prev Med 2003; 36(5): 615-23.

19. Fine JL, Philogene S, Gramling R, Coups EJ, Sinha S. Prevalence of Multiple Chronic Disease Risk Factors 2001 National Health Interview Survey. Am J Prev Med 2004; 27(2 Supl): 18-24.

20. Santos Filho RD, Martinez TLR. Fatores de risco para doença cardiovascular: Velhos e Novos Fatores de Risco, Velhos Problemas! Unidade Clínica de Dislipidemias, InCor, HCFMUSP e Departamento de Aterosclerose, Sociedade Brasileira de Cardiologia. Arq Bras Endocrinol Metabol 2002 jun; 46(3): 212-4.

21. Barnes PM, Adams PF, Schiller JS. Summary health statistics for the U.S. Population: National Health Interview Survey, 2001. [monografia na Internet]. Vital Health Stat 10. 2003 [acesso em: 2008 nov 26]. Disponível em http:// www.ncbi.nlm.nih.gov/pubmed/15791895.

22. Malta DC, Cezario AC, Moura L, Moraes Neto OL, Silva Junior, JB. A construção da vigilância e prevenção das doenças crônicas não transmissíveis no contexto do Sistema Único de Saúde. Epidemiologia e serviços de sau'de: revista do Sistema Unico de Sau'de do Brasil, 2006 set; 15(3): 47-65. 
23. Instituto Nacional de Alimentação e Nutrição (Brasil). Pesquisa nacional sobre saúde e nutrição: perfil de crescimento da população brasileira de 0 a 25 anos. Brasília, 1990.

24. Instituto Nacional do Câncer (Brasil). Prevalência de tabagismo no Brasil. Dados dos inquéritos epidemiológicos em capitais brasileiras, 2004 [monografia na Internet]. Rio de Janeiro-RJ: INCA. Disponível em: bvsms.saude.gov.br/ bvs/publicacoes/tabaco_inquerito_nacional_070504.pdf.

25. Sociedade Brasileira de Hipertensão [acesso em: 2008 nov 26]. Disponível em www.sbh.org.br. 


\section{Abstract}

In Brazil, due to changes in population demographic, epidemiological and nutritional profiles as well as the control achieved in a number of communicable diseases, in recent decades an inversion in the epidemiological profile, with a decrease in infectious diseases and a surge in the prevalence of Non Communicable Diseases (NCDs). There is therefore a need to study social determinants and risk factors for chronic and degenerative diseases in the country. The present study intended to assess the prevalence of exposure to major risk factors for NCDs of the Brazilian population. Systematic review of articles in Portuguese published between 2003 to 2008 in the scientific literature, identified through the online databases Latin American and Caribbean Health Sciences Literature (Lilacs) and International Health Sciences Literature (Medline) was done. Prevalence of risk factor exposure varied according to defined criteria and characteristics of the studied population. The following minimum and maximum prevalence values for each studied risk factor were found: smoking $8.7 \%$ to $28.8 \%$, alcohol abuse $0.1 \%$ to $37.7 \%$, excess weight $1.5 \%$ to $49.0 \%$, obesity $9.4 \%$ to $17.6 \%$, physical inactivity $20.1 \%$ to $43.1 \%$, hypertension $5.3 \%$ to $34.0 \%$, and diabetes mellitus $2.7 \%$ to $7.8 \%$. Variation in the degree of exposure to risk factors for NCDs in the population identified in the various studies points out the need to standardize measurement tools so that results from different sites can be compared. Moreover, the high prevalence of exposure to risk factors observed in some regions suggests that immediate responses by means of implementing strategies for prevention and healthcare promotion are necessary to reduce the Brazilian population exposure to risk factors associated to chronic diseases development.

Key words: Chronic Disease; Risk Factors; Prevalence; Morbidity Surveys; Cross-Sectional Studies; Brazil

\section{Resumen}

En Brasil, debido a cambios en los perfiles demográficos, epidemiológicos y nutricionales de la población y al control logrado en una serie de enfermedades transmisibles, se ha observado en las últimas décadas un cambio del perfil epidemiológico de la reducción de las enfermedades infecciosas y lo aumento significativo en la prevalencia de las enfermedades crónicas no transmisibles (ECNT). Este cambio justifica lo estudio de los factores de riesgo y determinantes sociales de las enfermedades crónico-degenerativas en el país. Este trabajo tuvo como objetivo conocer la prevalencia de la exposición de la población a los principales factores de riesgo para el desarrollo de las ECNT en Brasil. Se realizó una revisión sistemática de los artículos publicados en la literatura científica, a partir de las bases de datos online Lilacs y Medline, entre 2003 y 2008, en portugués. Las tasas de prevalencia variaron según los criterios utilizados y las características de las poblaciones estudiadas, y se obtuvo los siguientes valores mínimos y máximos para cada uno de los factores de riesgo analizados: consumo de tabaco $8,7 \%$ a $28,8 \%$, abuso de alcohol $0,1 \%$ a $37,7 \%$, sobrepeso $1,5 \%$ a $49,0 \%$, obesidad $9,4 \%$ al $17,6 \%$, sedentarismo $20,1 \%$ a $43,1 \%$, hipertensión arterial $5,3 \%$ a $34,0 \%$, diabetes mellitus $2,7 \%$ a $7,8 \%$. La variación en el grado de exposición de la población a los factores de riesgo presentes en los diversos estudios apunta para la necesidad de la estandarización de los instrumentos de medición, de modo que los resultados obtenidos en las diferentes localidades se puedan comparar. Además, la alta prevalencia observada en algunas zonas indica la necesidad de intervención inmediata a través de la aplicación de estrategias de prevención y promoción de la salud, dirigidas a reducir la exposición de la población brasileña a los factores asociados al riesgo del desarrollo de las ECNT.

Palabras clave: Enfermedad Crónica; Factores de Riesgo; Prevalencia; Encuestas de Morbilidad; Estudios Transversales; Brasil 